\title{
Design of Matched Absorbing Layers for Surface Plasmon-Polaritons
}

\author{
Sergio de la Cruz, ${ }^{1}$ Eugenio R. Méndez, ${ }^{1}$ and Alexei A. Maradudin ${ }^{2}$ \\ ${ }^{1}$ División de Física Applicada, Centro de Investigación Científica y de Educación Superior de Ensenada, \\ Carretera Ensenada-Tijuana No. 3918, 22860 Ensenada, BC, Mexico \\ ${ }^{2}$ Department of Physics and Astronomy and Institute for Surface and Interface Science, University of California, Irvine, \\ CA 92697, USA
}

Correspondence should be addressed to Eugenio R. Méndez, emendez@cicese.mx

Received 29 June 2012; Accepted 23 July 2012

Academic Editor: Natalia M. Litchinitser

Copyright ( $) 2012$ Sergio de la Cruz et al. This is an open access article distributed under the Creative Commons Attribution License, which permits unrestricted use, distribution, and reproduction in any medium, provided the original work is properly cited.

\begin{abstract}
We describe a procedure for designing metal-metal boundaries for the strong attenuation of surface plasmon-polaritons without the introduction of reflections or scattering effects. Solutions associated with different sets of matching materials are found. To illustrate the results and the consequences of adopting different solutions, we present calculations based on an integral equation formulation for the scattering problem and the use of a nonlocal impedance boundary condition.
\end{abstract}

\section{Introduction}

In the numerical solution of spatially unbounded electromagnetic problems it is often necessary to truncate the computational domain. The use of a region of finite spatial size can introduce reflections and other spurious effects in the calculations. Several techniques have been proposed to overcome this problem. An established one, used commonly in finite-difference time-domain (FDTD) calculations [1], is the perfectly matched layer (PML) technique proposed by Berenger [2]. Such a layer can absorb electromagnetic waves without reflections at the vacuum-PML interfaces. Methods of calculation based on Green's theorem (see, e.g., [3]) do not present such complications with volume waves, but the truncation of the interfaces can produce spurious effects in the presence of surface waves, like surface plasmonpolaritons (SPPs).

The basic properties of SPPs have been known for some time, but their importance for nanophotonic applications has produced a renewed interest on the subject $[4,5]$. In studies of the interactions of SPPs with objects or surface structures, the computational problem grows as a function of the physical size of the sample and, thus, it is desirable to reduce the computational domain as much as possible. The
PML techniques known to us were not designed to handle truncation effects involving SPPs, and are not well-adapted for situations involving metallic structures and evanescent waves [6].

In this paper, we present a procedure for determining the optical constants of absorbing materials for the attenuation of SPPs without introducing, or minimizing at least, spurious reflections and/or radiative scattering effects. Although SPPs are already lossy traveling waves, a reduction of their propagation length in the matched medium permits an important reduction in the dimensions of the region over which the computational domain extends. Although our approach is related in spirit to the usual PML techniques, we point out that it addresses a different problem, namely, the termination of surfaces over which surface waves propagate without the introduction of artificial scattering or reflection effects.

The absorbing materials that we propose are homogeneous and isotropic. The flexibility for choosing their optical properties permits the selection of materials that are appropriate for the use of impedance boundary conditions. All these facts simplify considerably the electromagnetic problem. The results presented here can be applied not only for electromagnetic calculations based on integral equations, 


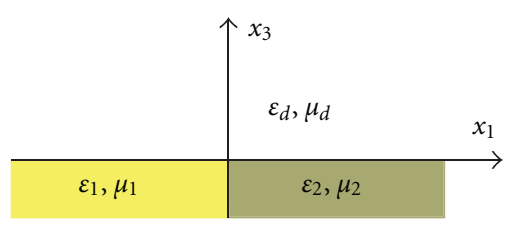

Figure 1: Schematic diagram of the three media considered. The upper medium is a dielectric, characterized by its electric permittivity $\epsilon_{d}$ and magnetic permeability $\mu_{d}$ in the frequency region of interest. Similarly, the metals 1 and 2 are characterized by $\epsilon_{1}, \mu_{1}$ and $\epsilon_{2}, \mu_{2}$, respectively.

like the one used for our examples, but also for those based on differential equations, like the FDTD.

\section{Theory}

To describe the technique, let us consider the generic geometry illustrated in Figure 1. The figure shows a flat interface involving three media: a semi-infinite dielectric and two metals, defined through their permittivities and permeabilities, with a vertical boundary. We assume that SPPs traveling from left to right, impinge on the vertical boundary between media 1 and 2. The optical properties of medium 2 are to be chosen in such a way that the SPPs are strongly attenuated without the introduction of significant reflections or coupling into radiative modes.

Let us consider the magnetic field associated with an SPP traveling on the flat surface of medium 2 , in the direction $+x_{1}$. This field may be conveniently written in terms of the magnetic field along the $x_{2}$-direction. We write

$$
\begin{array}{ll}
H_{2}^{>}\left(x_{1}, x_{3}\right)=H_{0} e^{i k_{s p}^{(2)} x_{1}+i \alpha_{d}\left(k_{s p}^{(2)}\right) x_{3}} & x_{3} \geq 0, \\
H_{2}^{<}\left(x_{1}, x_{3}\right)=H_{0} e^{i k_{s p}^{(2)} x_{1}-i \alpha_{2}\left(k_{s p}^{(2)}\right) x_{3}} & x_{3} \leq 0,
\end{array}
$$

where $H_{0}$ is a constant,

$$
\begin{gathered}
k_{s p}^{(2)}=\frac{\omega}{c} \sqrt{\frac{\epsilon_{d} \epsilon_{2}\left(\epsilon_{2} \mu_{d}-\epsilon_{d} \mu_{2}\right)}{\epsilon_{2}^{2}-\epsilon_{d}^{2}},} \\
\alpha_{d}\left(k_{s p}^{(2)}\right)=\sqrt{\epsilon_{d} \mu_{d}\left(\frac{\omega}{c}\right)^{2}-\left(k_{s p}^{(2)}\right)^{2}}, \\
\alpha_{2}\left(k_{s p}^{(2)}\right)=\sqrt{\epsilon_{2} \mu_{2}\left(\frac{\omega}{c}\right)^{2}-\left(k_{s p}^{(2)}\right)^{2}} .
\end{gathered}
$$

The signs of the square roots are taken in such a way that the imaginary parts of $k_{s p}^{(2)}, \alpha_{d}\left(k_{s p}^{(2)}\right)$, and $\alpha_{2}\left(k_{s p}^{(2)}\right)$, are positive. In the dielectric, the attenuation constant along $x_{3}$ is given by

$$
\kappa_{2}^{>}=\mathfrak{I} m\left\{\alpha_{d}\left(k_{s p}^{(2)}\right)\right\}=\frac{\omega}{c} \mathfrak{I} m\left\{\sqrt{\frac{\epsilon_{d}^{2}\left(\epsilon_{2} \mu_{2}-\epsilon_{d} \mu_{d}\right)}{\epsilon_{2}^{2}-\epsilon_{d}^{2}}}\right\} .
$$

Similar expressions can be written for the interface between the dielectric and medium 1 .
To minimize the scattering losses due to the vertical metallic interface of Figure 1, the transverse profile of the SPP wave must be the same on the two sides of the interface [7-9]. That is, one must have that $\kappa_{1}^{>}=\kappa_{2}^{>}$. On the other hand, the reflection of the SPP at the boundary should be minimized when $\mathfrak{R} e\left\{k_{s p}^{(2)}\right\}=\mathfrak{R} e\left\{k_{s p}^{(1)}\right\}$. We will refer to these conditions as the no scattering and no reflection conditions.

At this stage, we focus our attention on the case in which $\epsilon_{d}=1, \mu_{d}=1$, and assume that the metal on the left is nonmagnetic at optical frequencies (i.e., $\mu_{1}=1$ ), while that on the right has arbitrary properties. As a particular example, we consider the design of absorbing layers that match the properties of gold at the vacuum wavelength $\lambda=980 \mathrm{~nm}$. Then, $\epsilon_{1}=-40.44+i 2.97[10]$ and $\mu_{1}=1.0$. Since $\epsilon_{2}$ and $\mu_{2}$ are complex quantities, one must seek solutions in a fourdimensional space. To simplify matters, we have decided to fix the imaginary parts of $\epsilon_{2}$ and $\mu_{2}$, and explore the space $\left(\mathfrak{R} e\left\{\epsilon_{2}\right\}-\mathfrak{R} e\left\{\mu_{2}\right\}\right)$.

For physical reasons, we choose $\mathfrak{I} m\left\{\epsilon_{2}\right\}>0$. Although there seems to be no problem with the choice of a negative $\mathfrak{I} m\left\{\mu_{2}\right\}$ [11], we also took it as positive. With these choices, the search space is restricted to the quadrant defined by the conditions $\mathfrak{R} e\left\{\epsilon_{2}\right\}<0$ and $\mathfrak{R} e\left\{\mu_{2}\right\}>0$.

For our first example we have set $\mathfrak{J} m\left\{\epsilon_{2}\right\}=3.0$ and $\mathfrak{I} m\left\{\mu_{2}\right\}=0.0$. The solutions to the equations for no scattering and no reflection are shown in Figure 2(a). We observe that the two curves practically coincide when $\mathfrak{R} e\left\{\epsilon_{2}\right\}<-10$ and that they gradually separate as $\mathfrak{R} e\left\{\epsilon_{2}\right\}$ approaches zero. It is thus advisable to seek solutions in the region $\Re e\left\{\epsilon_{2}\right\} \leq-10$.

One can see in Figure 2(a) that the pair of $x, y$ values $(-40.44,1)$, which corresponds to the properties of medium 1 , lie on the solution curve. This is due to the closeness between the chosen value of $\mathfrak{I} m\left\{\epsilon_{2}\right\}$ and that of $\mathfrak{I} m\left\{\epsilon_{1}\right\}$.

As one moves along these solution curves from left to right, the absorption coefficient of the SPPs, $\mathfrak{I} m\left\{k_{s p}^{(2)}\right\}$, increases. The behavior is illustrated in Figure 2(b). On the basis of these results, and since we are looking for solutions with a strong attenuation coefficient, we have chosen a value of $\mathfrak{R} e\left\{\epsilon_{2}\right\}=-10.0$, which leads to $\mathfrak{R} e\left\{\mu_{2}\right\}=0.19$ and an attenuation constant $\mathfrak{I} m\left\{k_{s p}^{(2)}\right\}=0.033 \mu m^{-1}$. We will refer to this example as case I.

Values of the real parts of $\epsilon_{2}$ and $\mu_{2}$ that result in higher attenuation coefficients are not appropriate for our purposes, as they produce a mismatch between the no scattering and no reflection conditions. Moreover, as can be inferred from the discussion in the following section, they lead to solutions that are more difficult to handle computationally.

For our second example, we chose $\mathfrak{I} m\left\{\epsilon_{2}\right\}=3.0$ and $\mathfrak{I} m\left\{\mu_{2}\right\}=10.0$. The solution map is shown in Figure 3(a), and the attenuation coefficient for SPPs is shown in Figure 3(b). We see that, with these parameters, the curves corresponding to the no scattering and no reflection conditions are fairly close, but differ by an increasing amount as the magnitude of $\mathfrak{R} e\left\{\epsilon_{2}\right\}$ decreases. The attenuation coefficient curves, on the other hand, are practically indistinguishable in this case. For this second example, we choose $\mathfrak{R} e\left\{\epsilon_{2}\right\}=-531.62$, and based on 


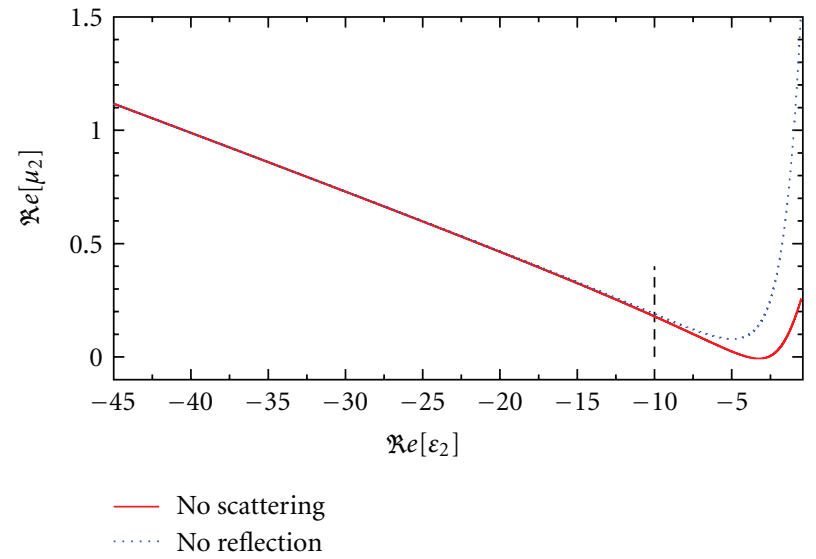

(a)

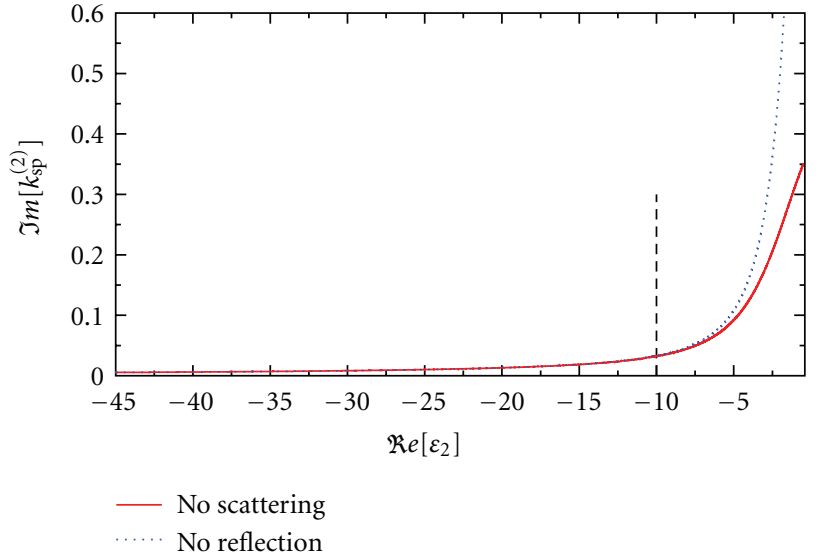

(b)

FIgURE 2: (a) Solution curves corresponding to the no reflection and no scattering conditions assuming $\mathfrak{I} m\left\{\epsilon_{2}\right\}=3.0$ and $\mathfrak{I} m\left\{\mu_{2}\right\}=0.0$. (b) Behavior of $\mathfrak{J} m\left\{k_{s p}^{(2)}\right\}$ along the solution curves. The value of $\mathfrak{R} e\left\{\epsilon_{2}\right\}$ chosen for the example is denoted by the vertical dashed line.

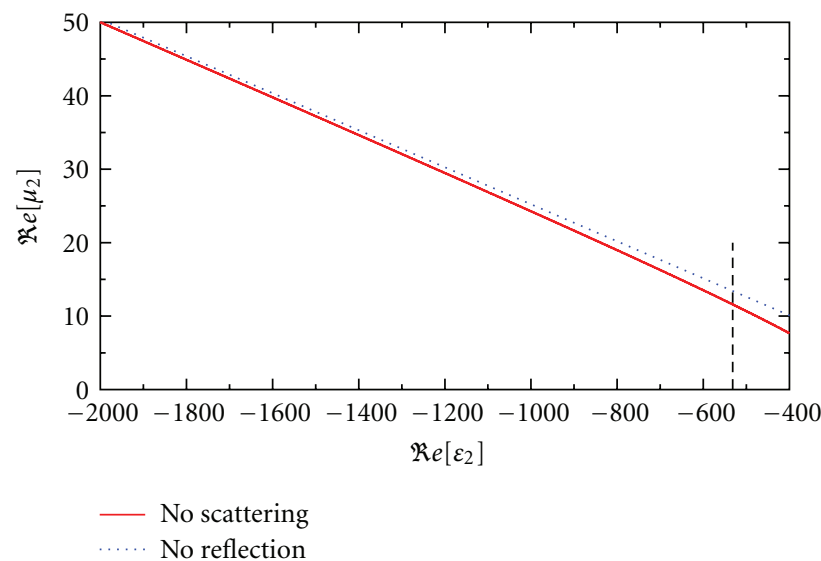

(a)

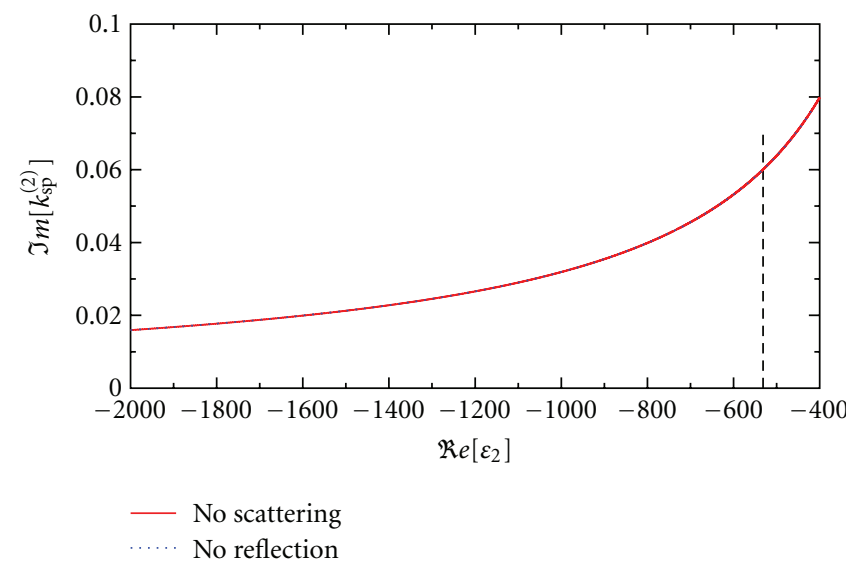

(b)

FIGURE 3: (a) Solution curves corresponding to the no reflection and no scattering conditions assuming $\mathfrak{I} m\left\{\epsilon_{2}\right\}=3.0$ and $\mathfrak{I} m\left\{\mu_{2}\right\}=10.0$. (b) Behavior of $\Im m\left\{k_{s p}^{(2)}\right\}$ along the solution curves. The value of $\mathfrak{R} e\left\{\epsilon_{2}\right\}$ chosen for the example is denoted by the vertical dashed line.

the no-scattering-condition curve, one has that $\mathfrak{R} e\left\{\mu_{2}\right\}=$ 13.44 and $\mathfrak{I} m\left\{k_{s p}^{(2)}\right\}=0.06 \mu \mathrm{m}^{-1}$. We will refer to this example as case II. It is worth mentioning that the results obtained with these parameters are practically the same as those obtained with the parameters corresponding to the no reflection curve.

Scattering calculations involving gold and the materials proposed in this section will be presented and discussed in Section 4.

\section{A Nonlocal Impedance Boundary Condition}

The attenuation of SPPs in the proposed matching media can be visualized through rigorous electromagnetic simulations of the problem. We base our calculations on an integral equation formulation that has been used for rough-surfacescattering studies [3]. Geometries like the one depicted in Figure 1 pose some difficulties for this kind of approach, but the problem can be simplified with the use of an impedance boundary condition [12-14]. The use of an impedance boundary condition in the present problem leads to a formulation of the scattering problem that does not require knowledge of the field below the interface.

Impedance boundary conditions have been used in the past for scattering calculations and are known to provide accurate results for good conductors, like gold and silver, in the near infrared [15]. We shall see however that, due to the rapid decay of the SPPs in the absorbing region, a local impedance boundary condition is insufficient to deal with some of the media considered here. So, before discussing the scattering calculations, we present a brief derivation of 
the impedance boundary condition for flat surfaces, which includes the first nonlocal term.

With reference to Figure 1, we consider the field below a flat interface, inside a medium with the properties of medium 2. It can be written in the form

$$
H_{2}^{<}\left(x_{1}, x_{3}\right)=\int_{-\infty}^{\infty} \frac{d q}{2 \pi} A(q) e^{i q x_{1}-i \alpha_{2}(q) x_{3}},
$$

where $A(q)$ is the scattering amplitude, or angular spectrum of the field below the interface.

Here, $\alpha_{2}(q)=\sqrt{\epsilon_{2} \mu_{2}(w / c)^{2}-q^{2}}$, with $\mathfrak{J} m\left\{\alpha_{2}(q)\right\}>0$.

Since

$$
A(q)=\int_{-\infty}^{\infty} d x_{1}^{\prime} H_{2}^{<}\left(x_{1}^{\prime}, 0\right) e^{-i q x_{1}^{\prime}}
$$

one can write

$$
\begin{aligned}
H_{2}^{<}\left(x_{1}, x_{3}\right)= & \int_{-\infty}^{\infty} \frac{d q}{2 \pi} \int_{-\infty}^{\infty} d x_{1}^{\prime} \\
& \times H_{2}^{<}\left(x_{1}^{\prime}, 0\right) e^{i q\left(x_{1}-x_{1}^{\prime}\right)-i \alpha_{2}(q) x_{3}} .
\end{aligned}
$$

From this expression, one can establish the following integral equation involving the field and its normal derivative on the interface:

$$
\left.\frac{\partial H_{2}^{<}\left(x_{1}, x_{3}\right)}{\partial x_{3}}\right|_{x_{3}=0}=\int_{-\infty}^{\infty} d x_{1}^{\prime} K\left(x_{1}, x_{1}^{\prime}\right) H_{2}^{<}\left(x_{1}^{\prime}, 0\right),
$$

where

$$
K\left(x_{1}, x_{1}^{\prime}\right)=\frac{1}{i} \int_{-\infty}^{\infty} \frac{d q}{2 \pi} \alpha_{2}(q) e^{i q\left(x_{1}-x_{1}^{\prime}\right)} .
$$

We now seek the solution of (7) by expanding $\alpha_{2}(q)$ as follows:

$$
\alpha_{2}(q) \approx \sqrt{\epsilon_{2} \mu_{2}}\left(\frac{\omega}{c}\right)\left[1-\frac{q^{2}}{2 \epsilon_{2} \mu_{2}(\omega / c)^{2}}+\cdots\right],
$$

which leads to

$$
\begin{aligned}
K\left(x_{1}, x_{1}^{\prime}\right)= & \frac{1}{i} \sqrt{\epsilon_{2} \mu_{2}}\left(\frac{\omega}{c}\right) \delta\left(x_{1}-x_{1}^{\prime}\right) \\
& +\frac{1}{2 i(\omega / c) \sqrt{\epsilon_{2} \mu_{2}}} \delta^{\prime \prime}\left(x_{1}-x_{1}^{\prime}\right)+\cdots .
\end{aligned}
$$

In this expression, $\delta\left(x_{1}\right)$ is a delta function and $\delta^{\prime \prime}\left(x_{1}\right)$ its second derivative.

Keeping only the first two terms, we find the relation

$$
\begin{aligned}
\left.\frac{\partial H_{2}^{<}\left(x_{1}, x_{3}\right)}{\partial x_{3}}\right|_{x_{3}=0}= & \left.\frac{1}{i} \sqrt{\epsilon_{2} \mu_{2}}\left(\frac{\omega}{c}\right) H_{2}^{<}\left(x_{1}, x_{3}\right)\right|_{x_{3}=0} \\
& +\left.\frac{1}{2 i \sqrt{\epsilon_{2} \mu_{2}}(\omega / c)} \frac{\partial^{2} H_{2}^{<}\left(x_{1}, x_{3}\right)}{\partial x_{1}^{2}}\right|_{x_{3}=0},
\end{aligned}
$$

and using the continuity of the tangential components of the fields across the interface we find that

$$
\begin{aligned}
\frac{1}{\epsilon_{d}} L^{>}\left(x_{1}\right)=\frac{1}{\epsilon_{2}} & {\left[\frac{1}{i} \sqrt{\epsilon_{2} \mu_{2}}\left(\frac{\omega}{c}\right) H^{>}\left(x_{1}\right)\right.} \\
& \left.+\frac{1}{2 i \sqrt{\epsilon_{2} \mu_{2}}(\omega / c)} \frac{d^{2} H^{>}\left(x_{1}\right)}{d x_{1}^{2}}\right],
\end{aligned}
$$

where

$$
\begin{gathered}
H^{>}\left(x_{1}\right)=\left.H_{2}^{>}\left(x_{1}, x_{3}\right)\right|_{x_{3}=0}, \\
L^{>}\left(x_{1}\right)=\left.\frac{\partial H_{2}^{>}\left(x_{1}, x_{3}\right)}{\partial x_{3}}\right|_{x_{3}=0} .
\end{gathered}
$$

Finally, we write (12) in the form

$$
L^{>}\left(x_{1}\right)=K_{p}^{(0)} H^{>}\left(x_{1}\right)+K_{p}^{(2)} \frac{d^{2} H^{>}\left(x_{1}\right)}{d x_{1}^{2}},
$$

where

$$
\begin{gathered}
K_{p}^{(0)}=\frac{\omega}{i c} \epsilon_{d} \sqrt{\frac{\mu_{2}}{\epsilon_{2}}}, \\
K_{p}^{(2)}=\frac{\epsilon_{d} / \epsilon_{2}}{2 i \sqrt{\epsilon_{2} \mu_{2}}(\omega / c)} .
\end{gathered}
$$

The first term in the impedance expansion (14) represents a local relation between $L^{>}\left(x_{1}\right)$ and $H^{>}\left(x_{1}\right)$ (or between the $E$ and $H$ fields). The second term, represents the first nonlocal correction.

We see that as the attenuation of the SPPs becomes stronger, the second derivative of the field appearing in (14) becomes larger. Looking at the quantities entering the expression for $K_{p}^{(2)}$, we conclude that it is desirable to have media with a high refractive index $n_{2}=\sqrt{\epsilon_{2} \mu_{2}}$. For a more quantitative evaluation of this issue, we consider the ratio between the first two terms of the expansion. This ratio $\mathcal{R}$ constitutes an incomplete but, nevertheless, essential knowledge for establishing the validity of the local relation and the convergence of the expansion. Using the general form of the field associated with an SPP on a flat surface given by (1a), we find that

$$
\mathcal{R}=\left|\frac{K_{p}^{(2)}\left(d^{2} H^{>}\left(x_{1}\right) / d x_{1}^{2}\right)}{K_{p}^{(0)} H^{>}\left(x_{1}\right)}\right|=\frac{1}{2}\left|\frac{k_{s p}^{(2)}}{n_{2}(\omega / c)}\right|^{2},
$$

where $n_{2}$ is the complex refractive index of medium 2 .

In normal circumstances (i.e., when $\mu_{2}=1$ ), for a good conductor one has that $n_{2}$ is large and that $\left|k_{s p}^{(2)}\right| \approx(\omega / c)$. Thus, $\mathcal{R}$ is small, and the nonlocal term can be neglected. For instance, for a gold-vacuum interface and the wavelength considered, this ratio is $\mathcal{R}=0.0125$.

Turning our attention to the absorbing materials proposed in Section 2, for our first example (case I) this ratio turns out to be $\mathcal{R}=0.258$. It is clear that a local impedance boundary condition would not be accurate on the absorbing side of the boundary and that nonlocal correction terms are needed. This conclusion is supported by the numerical calculations presented in Section 4.

For case II, the ratio between the first two terms of expansion (14) is $\mathcal{R}=1.67 \times 10^{-5}$. Not surprisingly, the local impedance boundary condition is very accurate in this case. This case leads to parameters that are more convenient for our type of calculation. 


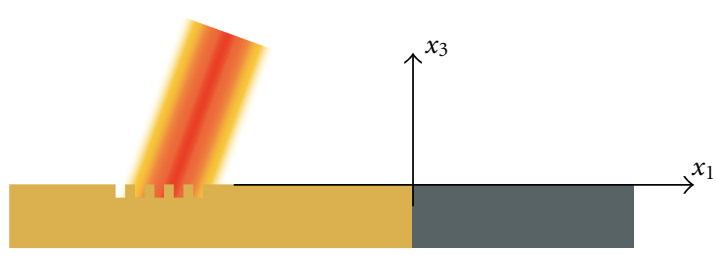

FIGURE 4: Schematic diagram of the scattering geometry considered for the calculations. A Gaussian beam illuminates a short gold grating that couples a fraction of the incident light to SPPs traveling to the right. The absorbing material is represented by the darker region.

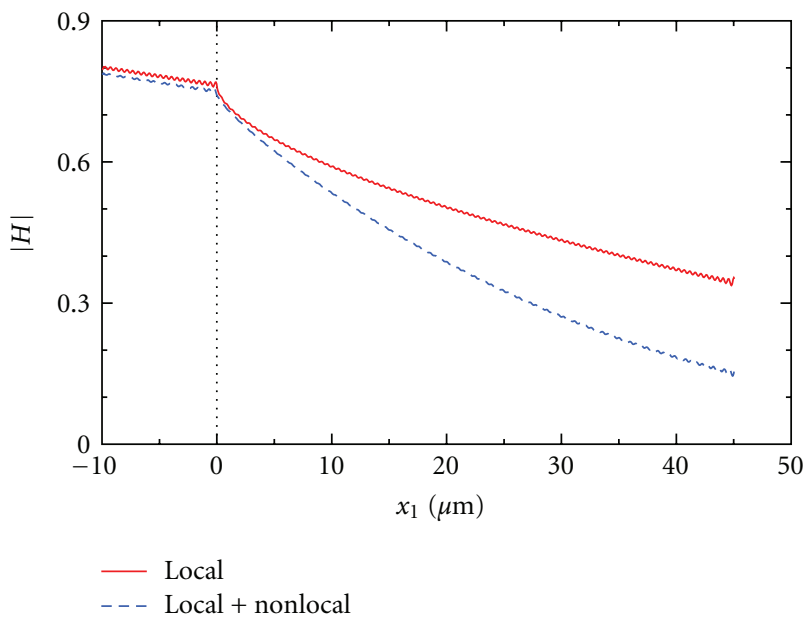

Figure 5: Magnitude of the surface field calculated using the local impedance boundary condition and the first nonlocal correction for case I. The boundary between the two metals is at $x_{1}=0$ and, to facilitate the visualization of the interaction of the SPP with the metal-metal boundary, the region of the grating is not shown.

\section{Results and Discussion}

In this section, we present scattering calculations corresponding to the two examples of matching media proposed in Section 2, starting with a summary of their properties: For case I we have $\epsilon_{2}=-10.0+3.0 i$ and $\mu_{2}=0.19$, while for case II, $\epsilon_{2}=-531.62+3.0 i$ and $\mu_{2}=13.44+10.0 i$. In the first case, the attenuation constant for SPPs is $\mathfrak{I} m\left\{k_{s p}^{(2)}\right\}=0.033 \mu \mathrm{m}^{-1}$, while for the second $\mathfrak{I} m\left\{k_{s p}^{(2)}\right\}=0.06 \mu \mathrm{m}^{-1}$.

The geometry employed for the scattering calculations is shown in Figure 4. Based on previous results [16], to excite SPPs traveling to the right, we use an array of 5 rectangular grooves of period $T=0.863 \lambda$, illuminated by a Gaussian beam of width $g=2 \lambda$ with an angle of incidence $\theta_{0} \approx-6.5^{\circ}$. The coupling efficiency for the SPPs travelling to the right is about $45 \%$. The distance between the start of the buffer material and the end of the surface is $45 \mu \mathrm{m}$.

The magnitude of the surface field associated with the SPP propagating along the surface for case I is shown in Figure 5. To simplify the visualization of the region of interest, the section with the grating was omitted from the figure. The curve shown with a continuous line was calculated with a local impedance boundary condition, while for the dashed-line curve we included also the first nonlocal term. One observes that the attenuation constant of SPP changes as the wave enters the absorbing material. As expected, the nonlocal correction does make a substantial difference in the calculations. The small ripples observed in the upper curve are due to standing waves caused by the reflection of the SPP at the end of the surface. With this material it is important to use at least the first nonlocal correction in expansion (14).

In Figure 6, we show the calculated magnetic near-field intensity map (i.e., $|H|^{2}$ ) for case I. As in the previous figure, only the rightmost section of the sample is shown. It can be observed that the SPP arrives from the left and decays quite steeply as it enters the absorbing medium. The results are encouraging. The incident SPP is not perturbed much by the interface, and it is clear that the reflection and scattering effects are low.

The near-field intensity map corresponding to case II is shown in Figure 7. The attenuation of the SPP is stronger in this case, and the local impedance boundary condition gives accurate results. As desired, the SPP wave practically vanishes after only a few microns. As in case I, the incident SPP does not appear to be perturbed much by the interface.

To investigate the possible leakage of the SPPs guided waves due to the absorbing boundary, we present, in Figure 8, far-field calculations corresponding to a complete gold sample and to samples terminated with the absorbing materials that we have called cases I and II (see Figure 4).

The scattering curves show that there is a relatively strong reflected beam in the specular direction $\left(\theta_{s} \approx-6.5^{\circ}\right)$ as well as a broad, grazing diffraction order. The rapid oscillations observed in the diffraction order for the case of the gold surface are due to the leakage of the SPP at the end of the surface, which interfere with the grazing order. Moreover, the SPP reflected at this end propagate back and leak into the specular direction upon interaction with the grating. This gives rise to the small-scale oscillations over the whole curve.

The differential reflection coefficient curves corresponding to cases I and II are much cleaner. In particular, the finescale oscillations that can be observed over the whole curve associated with the gold surface have disappeared. Also, the strong and rapid oscillations in the region of the diffraction order have been replaced by smoother and less important oscillations that seem to be due to residual scattering at the metal-metal interface.

\section{Summary and Conclusions}

In this paper, we have presented a procedure for designing metal-metal boundaries for the strong attenuation of surface plasmon-polaritons, minimizing the introduction of reflections or scattering effects.

We have illustrated the results by means of computer simulations based on an integral equation formulation of the scattering problem and an impedance boundary condition. Depending on the parameters chosen for the matching medium, nonlocal corrections might be needed for the calculations. 


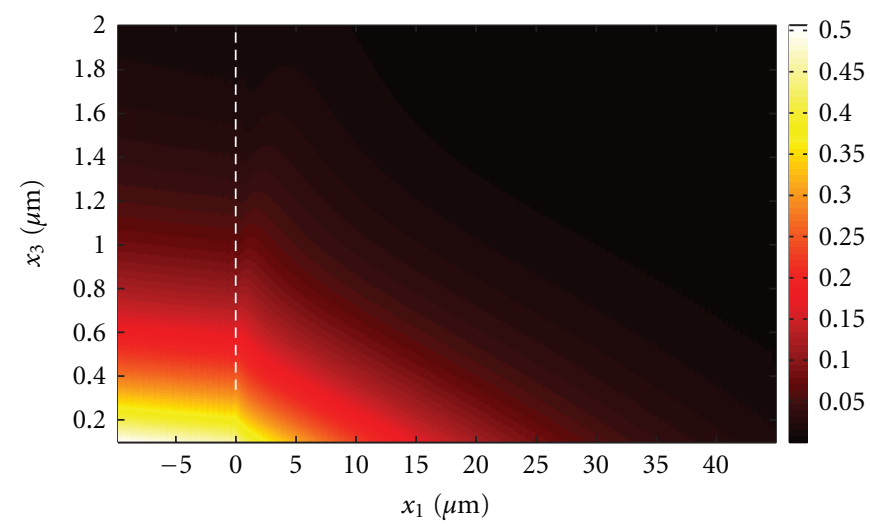

FIGURE 6: Squared modulus of the magnetic field in the vacuum region, above the flat metallic surfaces for case I. The position of the vertical border between the two metals at $x_{1}=0$ is denoted by the vertical dashed white line.

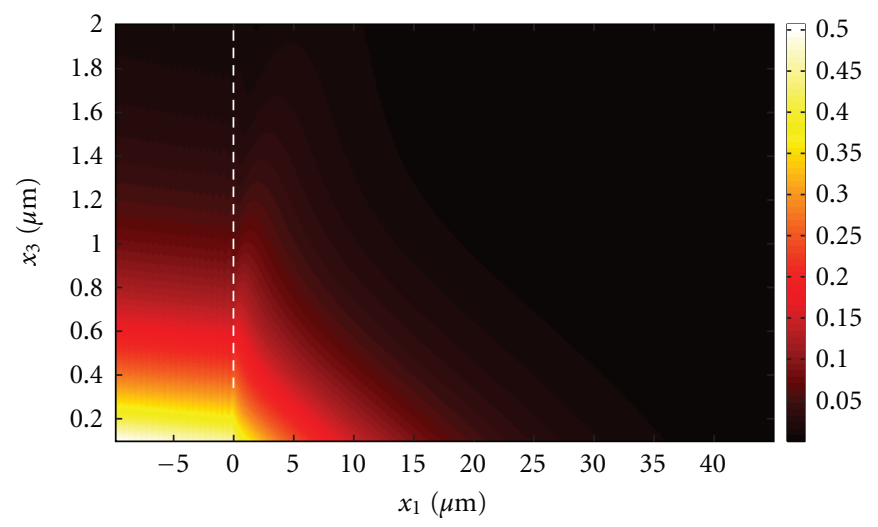

FIGURE 7: Squared modulus of the magnetic field in the vacuum region, above the flat metallic surfaces for case II. The position of the vertical border between the two metals at $x_{1}=0$ is denoted by the vertical dashed white line.

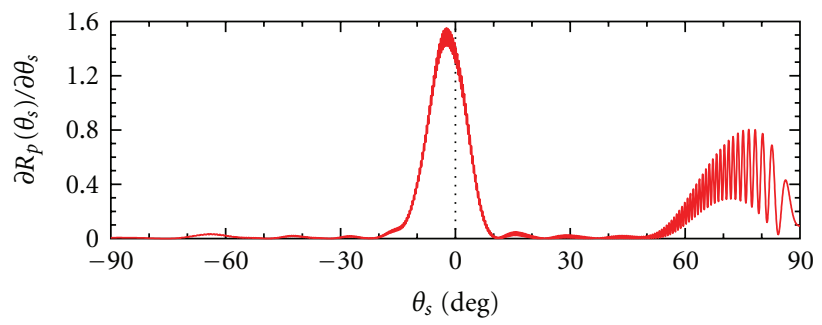

(a)

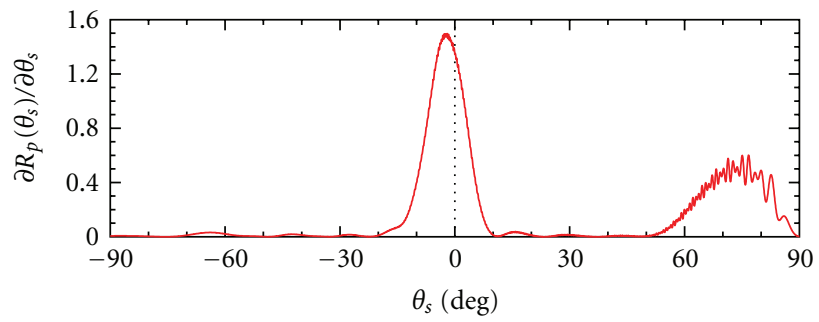

(b)

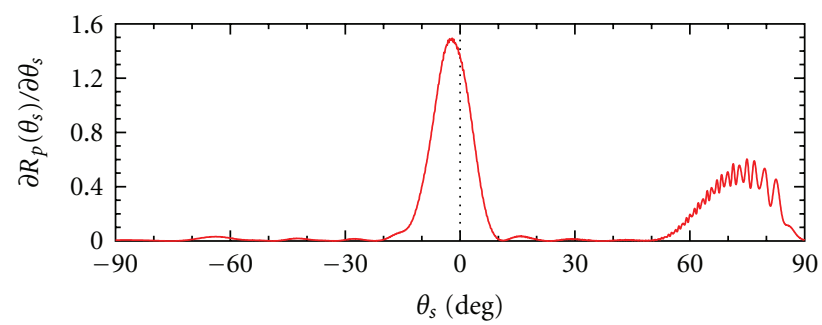

(c)

FIGURE 8: The differential reflection coefficient for a gold surface (a), a gold surface terminated with the material of case I (b), and a gold surface terminated with the material of case II (c). 
The results show that with the method described here, SPPs can be attenuated strongly without introducing significant effects in near field calculations. The propagation of the SPP is practically unperturbed along the original interface and spurious reflection and scattering effects are minimal. The implementation of the technique is fairly simple and can be used effectively in plasmonic and nanophotonic calculations by different methods.

The far-field results, however, seem to indicate the presence of some residual scattering originating from the metal-metal boundary. It is worth pointing out that this leakage is not due to the use of an impedance boundary condition or to the small difference between the values of the dielectric constants corresponding to the no-scattering and no-reflection conditions.

\section{Acknowledgments}

S. de la Cruz is grateful to the Consejo Nacional de Ciencia y Tecnología (CONACYT) for the award of a scholarship. The work of E. R. Méndez was supported in part by CONACYT, under Grant numbers 150466 and 180654. The research of A. A. Maradudin was supported in part by AFRL contract FA9453-08-C-0230.

\section{References}

[1] A. Taflove, Computational Electrodynamics: The FiniteDifference Time-Domain Method, Artech House, Boston, Mass, USA, 1995.

[2] J. P. Berenger, "A perfectly matched layer for the absorption of electromagnetic waves," Journal of Computational Physics, vol. 114, no. 2, pp. 185-200, 1994.

[3] A. A. Maradudin, T. Michel, A. R. McGurn, and E. R. Méndez, "Enhanced backscattering of light from a random grating," Annals of Physics, vol. 203, no. 2, pp. 255-307, 1990.

[4] A. V. Zayats, I. I. Smolyaninov, and A. A. Maradudin, "Nanooptics of surface plasmon polaritons," Physics Reports, vol. 408, no. 3-4, pp. 131-314, 2005.

[5] S. A. Maier, Plasmonics: Fundamentals and Applications, Springer, New York, NY, USA, 2007.

[6] C. C. Chao, S. H. Tu, C. M. Wang, H. I. Huang, C. C. Chen, and J. Y. Chang, "Impedance-matching surface plasmon absorber for FDTD simulations," Plasmonics, vol. 5, no. 1, pp. 51-55, 2010.

[7] R. F. Oulton, D. F. P. Pile, Y. Liu, and X. Zhang, "Scattering of surface plasmon polaritons at abrupt surface interfaces: implications for nanoscale cavities," Physical Review B, vol. 76, no. 3, Article ID 035408, 12 pages, 2007.

[8] J. Elser and V. A. Podolskiy, "Scattering-free plasmonic optics with anisotropic metamaterials," Physical Review Letters, vol. 100, no. 6, Article ID 066402, 4 pages, 2008.

[9] N. Zavareian and R. Massudi, "Study on scattering coefficient of surface plasmon polariton waves at interface of two metaldielectric waveguides by using G-GFSIEM method," Optics Express, vol. 18, no. 8, pp. 8574-8586, 2010.

[10] P. B. Johnson and R. W. Christy, "Optical constants of the noble metals," Physical Review B, vol. 6, no. 12, pp. 4370-4379, 1972.

[11] V. A. Markel, "Can the imaginary part of permeability be negative?” Physical Review E, vol. 78, no. 2, Article ID 026608, 5 pages, 2008 .
[12] R. A. Depine and J. M. Simon, "Surface impedance boundary condition for metallic diffraction gratings in the optical and infrared range," Optica Acta, vol. 30, no. 3, pp. 313-322, 1983.

[13] R. García-Molina, A. A. Maradudin, and T. A. Leskova, "The impedance boundary condition for a curved surface," Physics Report, vol. 194, no. 5-6, pp. 351-359, 1990.

[14] A. A. Maradudin and E. R. Méndez, "Theoretical studies of the enhanced backscattering of light from one-dimensional randomly rough metal surfaces by the use of a nonlocal impedance boundary condition," Physica A, vol. 207, no. 1-3, pp. 302-314, 1994.

[15] M. E. Knotts, T. R. Michel, and K. A. O’Donnell, "Comparisons of theory and experiment in light scattering from a randomly rough surface," Journal of the Optical Society of America A, vol. 10, no. 5, pp. 928-941, 1993.

[16] S. de la Cruz, E. R. Méndez, D. Macías, R. Salas-Montiel, and P. M. Adam, "Compact surface structures for the efficient excitation of surface plasmon-polaritons," Physica Status Solidi $B$, vol. 249, no. 6, pp. 1178-1187, 2012. 

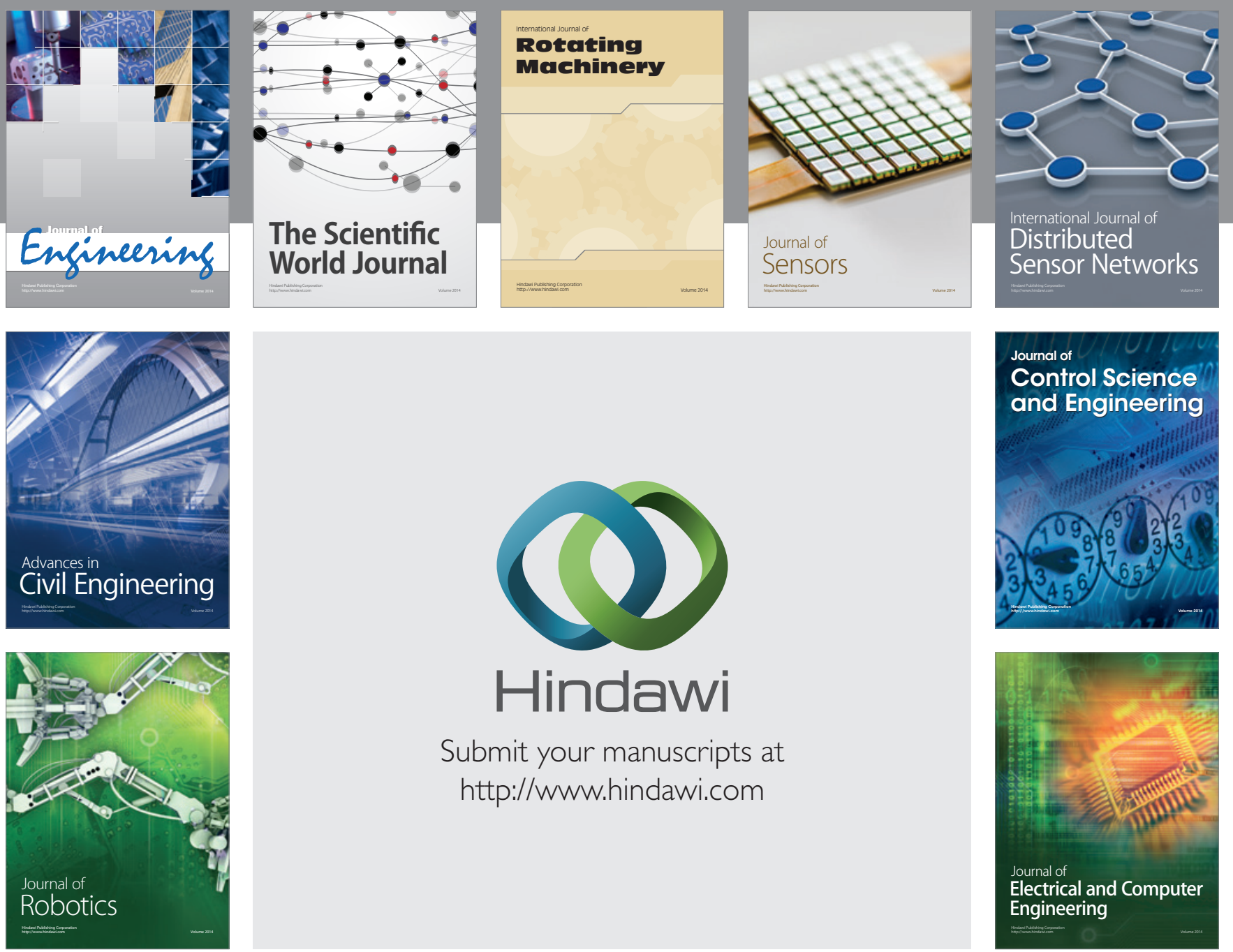

Submit your manuscripts at

http://www.hindawi.com
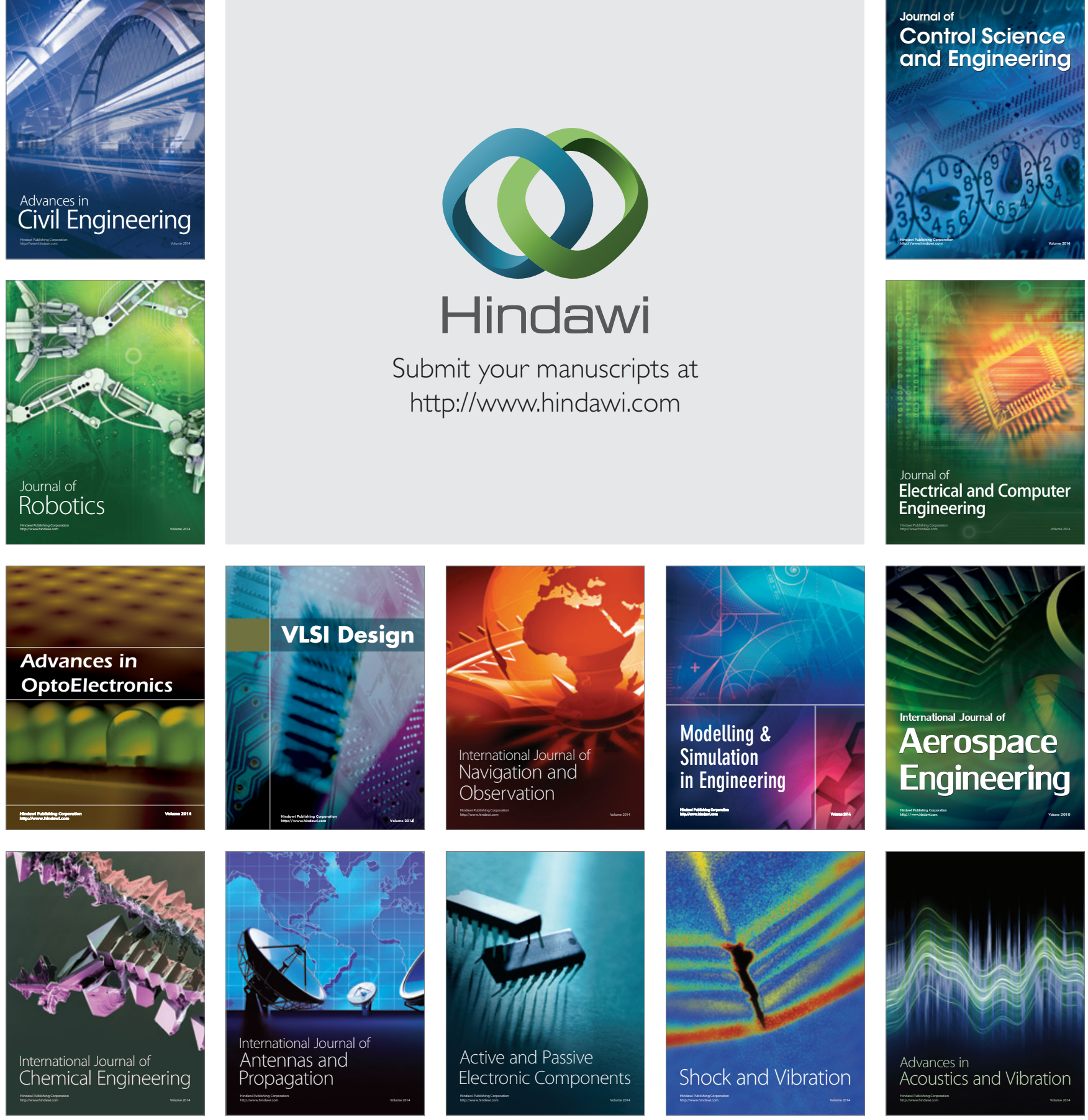